Voix et Images

voixetimages

\title{
Du Chemin Taché à la Vallée de Jonathan
}

\section{André Vanasse}

Volume 7, numéro 1, automne 1981

\section{Adrien Thério}

URI : https://id.erudit.org/iderudit/200302ar

DOI : https://doi.org/10.7202/200302ar

Aller au sommaire du numéro

Éditeur(s)

Les Presses de l'Université du Québec

ISSN

0318-9201 (imprimé)

1705-933X (numérique)

Découvrir la revue

Citer cet article

Vanasse, A. (1981). Du Chemin Taché à la Vallée de Jonathan. Voix et Images, 7(1), 35-43. https://doi.org/10.7202/200302ar d'utilisation que vous pouvez consulter en ligne.

https://apropos.erudit.org/fr/usagers/politique-dutilisation/ 


\title{
Du Chemin Taché à la Vallée de Jonăthan
}

\author{
André Vanasse
}

Écrire au Québec relève de l'héroïsme. Ou peut-être même de la folie. Comment peut-on imaginer que des hommes et des femmes puissent consacrer leur vie à une activité qui ne leur procurera que des déboires ou, au mieux, un succès d'estime?

J'admire les professionnels de l'écriture. Je songe plus particulièrement à Gabrielle Roy, à Marie-Claire Blais. Elles ont sacrifié vingt ans, trente ans de leur existence pour constater que le public ne connaît d'elles que Bonheur d'occasion ou Une saison dans la vie d'Emmanuel.

Encore faut-il se rappeler qu'elles ont été couronnées d'un prix étranger. La première d'un Fémina, la seconde d'un Médicis. Pour les autres écrivains, l'indifférence est leur lot. Surtout s'ils ratent le circuit scolaire (celui dont ont bénéficié les Yves Thériault, Jacques Godbout, Gérard Bessette).

Tel est le sort qu'a connu Adrien Thério. Pour fêter ses vingt-cinq ans de vie d'écrivain, il avait produit $C$ 'est ici que le monde a commencé, un roman d'une indéniable qualité. Résultat: ụne ou deux critiques dans des journaux de l'extérieur de Montréal. Silence dans les grands quotidiens.

J'imagine aisément la blessure que cet «oubli» peut causer. Une vie pour aboutir à l'impasse. De quoi assommer le plus optimiste.

Pourtant Adrien Thério continue son œuvre. Pionnier des lettres québécoises, il a été l'un de ceux qui ont le plus fait pour la diffuser. A l'heure où les intellectuels s'alimentaient aux comptoirs français, il se vouait à la défense et à l'illustration de notre littérature. En 1961, il créait Livres et A uteurs québécois, l'une des premières revues (avec les Écrits du Canada français et Liberté) à se consacrer exclusivement à nos auteurs. La revue dure toujours. Elle est entre les mains de l'Université Laval.

En 1976, malgré qu'il eût perdu une petite fortune dans Livres et Auteurs québécois, Adrien Thério lançait, avec autant d'enthousiasme, Lettres québécoises, un magazine de l'actualité littéraire. II allait connaître les mêmes soucis financiers. Mais cela est connu de la communauté intellectuelle québécoise. Ce qui étonne par contre, c'est le silence qui entoure son travail 
d'écrivain. Bien sûr un directeur de revue n'est pas nécessairement un bon auteur. Encore faudrait-il qu'on ait lu son œuvre. Il semble malheureusement que ce ne soit pas le cas.

Qui connaît La Colère du père, Soliloque en hommage à une femme, Les Fous d'amour, C'est ici que le monde a commencé? Ce sont pourtant des cuvres fortes qui ne méritent pas l'oubli dans lequel on les a plongées. J'allais passer sous silence Les Brèves Années, le premier roman de Thério que j'ai relu avec étonnement. C'est un livre touchant. Une matrice aussi : il y a, dans ce premier essai, la matière et le ton de ceux qui vont suivre.

Car on n'y échappe pas. La réitération (ou la compulsion de répétition) constitue à la fois le piège et la signature de tout véritable écrivain. C'est en revenant, parfois jusqu'à l'obsession, sur les mêmes images qu'il parvient à leur donner un degré de profondeur inégalée.

\section{Le Chemin Taché}

Nous sommes tous prisonniers de notre enfance. Cette assertion est encore plus vraie pour l'écrivain. Thério n'échappe pas à la règle. Le Chemin Taché, c'est-à-dire le coin de pays, près de Rimouski, où il est né, sillonne de part en part ses écrits. II y revient sans cesse peut-être pour déchiffrer une énigme dont il chercherait en vain la solution. "C'est ici que le monde a commencé » disait le titre de son dernier roman. On s'étonne alors qu'il le fuie comme un hors-la-loi. Adrien Thério a enseigné en Ontario, en Colombiebritannique, dans l'Indiana, dans le Kentucky mais jamais au Québec. Éternel voyageur, lorsqu'il s'asseoit à sa table de travail, il retourne pourtant, comme malgré lui, au pays de ses ancêtres. II n'y peut rien. II promène le Chemin Taché comme un tatouage.

Mais qu'a-t-il de si particulier, ce pays de son enfance? A vrai dire rien de bien glorieux. Il ressemble, du moins je l'imagine, à tous les rangs de la Gaspésie. Quand Jacques Cartier pénétra en Amérique par le golfe SaintLaurent, il qualifia de «Terres de Caïn » les rives qui le bordaient. L'histoire devait lui donner raison. Ceux qui s'y établirent, connurent la pauvreté pour les siècles à venir.

C'est du reste un des éléments frappants des récits de Thério. Ses personnages souffrent dans leur chair et dans leur âme de leur situation. Comment un enfant peut-il oublier qu'il a été forcé de se rendre à l'école sans souliers? Ou, par la suite, d'y être allé avec des chaussures pointues si ridicules qu'il a été la risée du village. Comment accepter que, exilé à la ville pour y poursuivre ses études, il puisse recevoir de gaieté de cœur une lettre de ses parents lui annonçant qu'ils ne peuvent payer son voyage aller-retour pour les vacances de Noël. Il est des humiliations qu'on avale difficilement. Entre autres, celle d'être à la merci du bon vouloir de la paroisse, sans quoi aucunes études supérieures ne seraient possibles. Les narrateurs, dans plusieurs récits de Thério, ont vécu de la charité publique, de la charité ecclésiastique. Ils devaient 
payer de leur vocation religieuse les sommes qu'on avait investies en eux. Quand on n'a pas la vocation mais le désir intense de s'instruire le déchirement est grand.

II se pourrait fort bien que cette dépendance, que cette soumission obligée devant l'autorité soit la clef de voûte de toute son œuvre.

\section{L'autorité des pères}

On a beaucoup parlé de l'image de la mère dans la littérature québécoise. Le sujet est devenu une image d'Épinal: alors que le père fuyait ses responsabilités pour courir les bois ou les routes, la mère, gardienne du foyer, assumait seule les destinées de la famille.

II y a du vrai dans cette interprétation. Notre littérature présente une image fracturée du père. D'un point de vue socio-critique et psychanalytique, le père porte comme une fêlure sa situation de dominé. Parfois il se révolte comme Menaud mais rarement réussit-il à terrasser le conquérant. Éternel vaincu, il a chóisi d'errer comme le Canadien de la célèbre chanson. "Le malheureux qui porte dans son cœur un ennui naturel, s'il croit trouver toujours plus loin un remède à sa peine, c'est pour rien qu'il quitte sa maison, son pays, et qu'il erre de place en place. Partout, jusqu'à la tombe, il emportera avec soi son ennui " "dira Didace Beauchemin au Survenant en guise d'avertissement.

Le malheur, une certaine littérature s'est évertuée à nous le prouver, c'est qu'on y échappe difficilement.

A y regarder de plus près cependant, on doit bien admettre qu'il existe de multiples personnages de la trempe des Didace Beauchemin et des Menaud. Ceux-ci s'acharnent à perpétuer la lignée, à faire en sorte que leurs descendants conservent intacte la fierté de la race. Ce sont des pères à la poigne de fer dont les enfants, ironie du sort, subissent l'effet castrateur de leur suprême autorité. Voyez le pauvre Amable.

Chez Thério, l'autorité patriarcale écrase non seulement les enfants mais les femmes aussi. Ces dernières se taisent devant leur mari, craignant une colère dont les effets pourraient être dévastateurs. Dès Les Brèves Années on voit poindre l'image du père en colère. Le père Martin terrorise toute sa famille. Il cherche noise à tous mais surtout à ses enfants qui abordent le seuil de l'adolescence. Alors ils deviennent véritablement des souffre-douleur grâce à la complicité des mères silencieuses et terrorisées. Ainsi, au début du roman, Pierre, l'aîné de la famille Martin, subit à tout propos les foudres de son père. Incapable de résister à tant de malveillances, il s'enfuit un jour au NouveauBrunswick. Puis ce sera au tour de Clair, devenu adolescent, d'être l'objet des récriminations paternelles.

Le père Martin, cela est évident, se sent menacé dans son autorité. Redoutant d'être supplanté par ses fils, il prend les devants et tente de les mater avant qu'il ne soit trop tard. 
Le meurtre du père n'aura pas lieu. La horde sauvage, dont a parlé Freud pour décrire la coalition des fils contre le père, ne pourra se former et mener à terme son projet parricide.

Par un étrange pouvoir de mimétisme, Clair introjectera le comportement paternel. Au collège Saint-Janvier où il a été placé, il se montrera à ce point retors devant l'autorité qu'il devra de lui-même quitter l'institution avant la fin de l'année. "Tel père, tel fils" nous dit l'adage. A cette différence près cependant que l'attitude de Clair apparaîtra plus positive que celle du père aux yeux du narrateur.

En réalité cette apparente contradiction se perpétue d'une œuvre à l'autre. II n'y a qu'une façon de rivaliser avec les pères honnis, c'est de leur voler leur propre pouvoir par le relais du mimétisme. Les héros de Thério sont tous des Prométhée enchaînés. Les pères comme les fils. Car si les fils sont soumis à la dure loi du père, les pères, eux, tout dictateurs qu'ils soient à l'intérieur de la cellule familiale, doivent subir les décrets de l'institution religieuse (laquelle se confond, dans la situation présente, avec l'institution sociale).

Le récit intitulé La Colère du père illustre fort bien cette lutte hiérarchique. Gaudiose sème la terreur dans sa maisonnée. II prend toute la place. Au point où son absence crée l'angoisse. Le narrateur nous confie à ce sujet: "Je redoutais mon père quand il piquait ses colères mais il n'en restait pas moins que c'était lui qui remplissait toute la maison quand il était là. Même quand il s'absentait, il suffisait de savoir qu'il allait rentrer d'une minute à l'autre (...) pour que son ombre se projette partout ${ }^{2}$.»

Or ce père, nulle part et partout à la fois, sorte de dieu redoutable, est lui-même dépendant de l'autorité ecclésiastique. II doit plier l'échine quand Julius, l'évêque de Rimouski, lui ordonne, par la voix du curé Saint-Onge, de se soumettre à sa volonté et d'accepter que l'église du rang, construite de ses mains, soit abandonnée au profit d'une nouvelle qui serait mieux située par rapport à l'ensemble de la population de la paroisse Saint-Hubert.

Gaudiose se révolte. Il se rend même à Rimouski pour dire son fait à ce Julius Marchildon, «gros plein de soupe ${ }^{3}$ » et prétentieux évêque. Rien n'y fait.

Alors, fou de colère, il entraîne ceux du Chemin Taché dans une aventure pour le moins inattendue. II décide de fonder une église protestante et trouve le moyen de faire venir de Montréal le pasteur Livingston pour qu'il prenne en charge les destinées des habitants du Chemin Taché.

Or ce pasteur, tout à fait charmant au demeurant, connaîtra lui aussi "la colère du père». II aura eu le malheur de s'amouracher de Claudia, la fille de Gaudiose. La réaction de ce dernier sera conforme à son caractère: «Prêtre ou pasteur, c'est la même chose. Y ont pas le droit de se marier ${ }^{4} . »$ «Ce mariage-là se fera famais, Obéline! (...) Si y faut mettre le pasteur à la porte, on va le mettre à la porte ${ }^{5}$." 
Et c'est ce qui se produira. Le pasteur Livingston quittera le Chemin Taché “pour des raisons personnelles ${ }^{6}$.

Gaudiose, on le voit, agit avec tous en contestataire et en tyran. II n'accepte aucune forme d'autorité, d'où qu'elle vienne. Il veut imposer par la force le respect de ses décisions arbitraires. II en viendra même aux coups avec Dieudonné (quel nom prédestiné!), son fils aîné, quand celui-ci, rendu à bout par les excès de son père, s'interposera entre son père et sa mère: Gaudiose a interdit à sa femme, Obéline, de prier! II sera maté par son fils.

Cuisante humiliation qui couronne la série de défaites de Gaudiose.

On ne saura jamais pourtant si les gens du Chemin Taché retourneront à leur ancienne foi. Le dernier chapitre se présente sous la forme d'un rêve au cours duquel Gaudiose “lance la chapelle sur un mur de pierres ${ }^{7}$.

Une autre sainte colère!

\section{Contestation et anticonformisme}

II y a, entre Les Brèves Années et La Colère du père, une différence essentielle. Dans le premier récit, le père Martin se servait de la religion pour mieux asseoir son autorité. Son orgueil, comme il est dit, c'était

l'orgueil du chef de famille conscient de sa souveraineté, qui doit exercer son autorité comme étant le représentant de Dieu et ne pas permettre un seul petit accroc à ce pouvoir même s'il était justifié par les circonstances. (...)

C'était aussi l'orgueil d'élever ses enfants dans «la crainte et l'amour de Dieu "comme disait lui-même le père Martin.

(...) II oubliait volontiers «l'amour de Dieu $* 8$.

Or dans La Colère du père, récit postérieur d'une dizaine d'années aux Brèves Années, le rapport au phénomène religieux s'est considérablement modifié. II se peut que ce changement d'attitude soit lié à l'évolution socioculturelle des Québécois des années soixante. Chose certaine, la religion est ici prise ouvertement à partie. Elle constitue une entrave, un joug contre lequel Gaudiose se révolte.

Ce dernier conteste d'abord et avant tout l'absolutisme du système religieux. Il le fait par principe, refusant d'être bêtement soumis à la volonté d'un autre, fût-ce celle d'un évêque. Il lui semble qu'il a, lui aussi, droit au chapitre. Que l'on n'ait pas cru bon de le consulter lui paraît une injure à sa dignité. II a raison. Voilà pourquoi, malgré son caractère irascible, Gaudiose nous demeure sympathique.

Pourtant sa révolte déçoit. Elle est spéculaire: la religion protestante contre la religion catholique. "OEil pour œil» disait la Bible. A ce prix, il n'y a pas de régénérescence possible. Les valeurs nouvelles véhiculées par la religion protestante, bien qu'elles soient perçues par Gaudiose, sont mises en veilleuse 
au profit de sa hargne. Le départ forcé du pasteur Livingston le confirme.

II n'en demeure pas moins que dans La Colère du père souffle un vent de contestation qui nous rafraîchit. À ce titre, les œuvres de Thério sont toujours volontairement anticonformistes. Elles récusent le pouvoir établi et les lois qui le régissent. Un paien chez les pingouins n'est qu'une autre facette de cette attitude. Le narrateur, cette fois-là, s'en prend à ces «pingouins» que sont les professeurs d'universités. Ce sont de ridicules et insignifiants personnages guindés dans la majesté factice de leur fonction.

Mais la plupart du temps les romans et récits de Thério s'attaquent à l'institution religieuse. II y a du Luther chez lui. Une sorte de plaisir secret à ébranler sur ses bases une Église figée dans ses préceptes.

Les Fous d'amour illustre de belle façon les procédés qu'il utilise pour miner l'Église de l'intérieur. L'action se déroule dans un monastère. Le narrateur, chroniqueur du monastère, consigne dans son «grand livre» les événements quotidiens qui marquent la vie de la communauté. Chroniques banales, sereines, pourrait-on croire puisque, dans un monastère, il ne devrait pas se produire de grands bouleversements. Tel n'est pas le cas. On y apprend qu' «à la date du 13 juillet de l'an dernier ${ }^{9}$ », les moines ont vécu un grand drame:

«Aujourd'hui le père Aurélien et le père Augustin se sont battus avec le père Armour. Ce dernier transporté à l'hôpital, a repris conscience et se remettra, d'après le médecin, de ses blessures qui sont moins graves qu'on ne l'avait cru d'abord ${ }^{10}$."

Ainsi Les Fous d'amour se présente comme le récit d'une dynamique de groupe. Le Père Armour, nouveau Christ du XXe siècle, connaîtra le sort qui fut réservé à celui qui lui servit de modèle: il sera ignominieusement assassiné dans le verger où il travaillait. Tout nous laisse croire qu'Aurélien est l'assassin. II subsiste cependant suffisamment de doute pour que nous puissions croire qu'en somme ce soit le groupe qui, par personne interposée, ait provoqué la mort du Père Armour.

Par certains côtés, le Père Armour ressemble au pasteur Livingston. Mais en mieux. II a pour lui toutes les qualités susceptibles de provoquer la jalousie:

Est-ce exagéré de dire que nous le détestions tous un peu, que nous l'enviions tous un peu, qu'intérieurement, sans nous en rendre compte, nous formions des vœux pour que Dieu rabaisse son orgueil? Mais qui me prouve qu'il y avait en lui de l'orgueil? Le Père Armour est beau, grand, bien fait d'épaule et de taille. On dirait que là où il va, il amène avec lui un vent de printemps, qu'il n'a qu'à faire un geste de la main pour faire disparaître les effets de la tristesse ou des nuages à l'horizon ${ }^{11}$.

Tout ce qu'il touche rayonne. Les hommes comme les plantes. Voilà pourquoi, à titre d'intendant de ferme et de maître des novices, il accomplit des miracles. Le «domaine est devenu une sorte de grand jardin ${ }^{12}$ » et il réussit "des merveilles chez (les) aspirants à la vie religieuse ${ }^{13}$ ". 
Les autres ne lui pardonnent pas son succès. D'autant plus que le Père Armour leur propose une vision hédoniste de la religion. Pour lui, il s'agit de rendre grâce à Dieu dans l'amour et dans la joie. Au Père Réjean qui lui affirme que «la vie d'un moine est une vie de renoncement et non de jouissance ${ }^{14}$ ", il réplique: "Donc je ne suis pas un bon moine puisque je jouis de tout ce que la nature peut m'offrir ${ }^{15}$."

Aux yeux du Père Armour, la religion est amour de la même façon qu'elle l'était pour le pasteur Livingston. Le Père Armour pousse jusqu'à l'extrême cette certitude. Chez lui aucune censure, aucune retenue. Un jour de chaleur intense, il permettra même aux membres de la communauté de se baigner nus dans le petit lac du domaine.

Inutile de dire que le laxisme du Père Armour bouleverse des habitudes séculaires. Plusieurs s'interrogent à la fois sur leur Supérieur (car il a été élu, contre toute attente, chef du monastère) mais aussi sur eux-mêmes. Ils s'éveillent à la vie, à leur corps, à leurs sens. Ils se retrouvent tout à coup dans une abbaye de Thélème réservée aux hommes: on leur propose une nouvelle manière de vivre, une nouvelle religion aussi, presque païenne...

On comprend alors pourquoi le Père Armour devra payer de sa vie la graine de folie qu'il a semée dans cette enceinte sacrée. Il a voulu modifier les règles du jeu, faire en sorte que la joie, mais aussi le désir, s'installent dans un lieu où, d'ordinaire, règne l'austérité. Sa jeunesse et son enthousiasme l'auront perdu. Sa punition sera plus terrible que celle du pasteur Livingston sans doute parce qu'il aura osé plus que lui.

\section{Désir et utopie}

Dans Les Fous d'amour, il faut le dire, règne une certaine ambiguïté. La «morale» du Père Armour semble suffisamment déviante pour laisser naître chez les moines d'évidents fantasmes homosexuels. Le désir circule en toute liberté. Certains laissent courir leur imagination à la vue de corps nus et beaux...

La baignade au lac a été la cause de ce débordement. II faut signaler, en passant, la récurrence de cette image dans plusieurs autres textes de Thério. Ainsi, dès Les Brèves Années, la même scène nous était présentée («nous courrions à la rivière, enlevions nos vêtements en un tour de main (sic) et sautions dans l'eau tiède en criant de joie ${ }^{16}$ ", puis dans Soliloque en hommage à une femme où le frère et la sœur vont connaître leur première relation incestueuse ( «Au moment où il t'apparaît dans sa nudité, tu acceptes de laisser tomber ce qui te reste de vêtements. (...) Vous vous sentez heureux de pouvoir nager ainsi sans que rien ne gêne vos mouvements ${ }^{17}$ " et finalement dans C'est ici que le monde a commencé où l'expérience prend véritablement la forme d'un rite initiatique.

Dans tous les cas, il s'agit d'un moment privilégié mais qui, d'un autre côté, pose quelques difficultés morales. Est-ce bien, est-ce mal? La réponse 
est claire: si c'est mal, alors au diable la morale!

En ce sens, C'est ici que le monde a commencé marque une rupture par rapport aux récits précédents. L'homosexualité toujours présente dans les autres écrits mais jamais totalement assumée, est ici officiellement reconnue. Pour être plus précis, il faudrait dire la bisexualité puisque l'homosexualité n'est pas exclusive.

En outre, les jeunes adolescents qui s'adonnent à leurs jeux «innocents" instaurent en même temps une nouvelle religion, décalque de la religion catholique à laquelle se mêlent des rites dionysiaques. Tout se passe comme si C'est ici que le monde a commencé proposait la réconciliation du religieux et du sexuel selon une hiérarchie où le religieux serait soumis à la fonction sexuelle.

Quant à la violence, élément caractéristique de l'œuvre de Thério, elle est canalisée dans la sexualité et non plus déviée de son but. Ainsi, quand le narrateur découvre la vallée de Jonathan, la vallée du commencement du monde par opposition à la vallée de Josaphat, il assiste à un spectacle grandiose «au cours duquel un grand cheval gris bleu, entouré d'une cohorte de géants, s'avance sur ses pattes d'arrière et prend possession du lieu en le noyant de son sperme qui devient une rivière où les gens vont se baigner. Et, dit le Père Ori: «J'ai même découvert une chose qui va te surprendre, c'est que ce beau cheval, c'est Dieu le Père. J'en suis sûr ${ }^{18}$."

Avec C'est ici que le monde a commencé, l'écriture de Thério débouche sur le mythique. Les fils devenus pères (cf. le pasteur Livingston, le Père Armour) avaient, dans les œuvres précédentes, été écrasés par les pères plus àgés. Ici, au contraire, le vieux Père Ori, contestataire comme les fils, permet au narrateur d'accéder à la vallée de Jonathan, «aux confins des terres du Chemin Taché ${ }^{19}$ » et de connaître enfin la révélation, de résoudre l'énigme qui poursuivait les narrateurs depuis Les Brèves Années.

Cette révélation qui ouvre la voie à une exploration du mythique me laisse croire que Thério a encore beaucoup à dire...

1. Germaine Guèvremont, Le Survenant, Montréal, Fides, «Nénuphar», 1968, p. 136.

2. Adrien Thério, La Colère du père, Montréal, Éd. Jumonville, 1974, pp. 15-16.

3. Ibid., p. 128.

4. Ibid., p. 161.

5. Ibid., p. 164.

6. Ibid., p. 175.

7. Ibid., p. 178.

8. Adrien Thério, Les Brèves Années, Montréal, Fides، «Alouette bleue», 1961, p. 41.

9. Adrien Thério, Les Fous d'amour, Montréal, Ed. Jumonville, 1973, p. 17.

10. Ibid., p. 17.

11. Ibid., p. 17. 
12. Ibid., p. 20.

13. Ibid., p. 24

14. Ibid., p. 23.

15. Ibid., p. 23

16. Adrien Thério, Les Brèves Années, p. 60.

17. Adrien Thério, Soliloque en hommage à une femme, Montréal, Le Cercle du livre de France, 1968 , p. 36.

18. Adrien Thério, C'est ici que le monde a commencé, Montréal, Éd. Jumonville, 1978, page couverture.

19. Ibid., page couverture. 